\title{
Performance Analysis of Quantized Feedback JLS Precoder in CoMP transmission
}

\author{
Yasmine Fahmy
}

\begin{abstract}
Joint Processing (JP) in Coordinated Multi-Point (CoMP) transmission allows user data to be jointly processed by several interfering base stations to achieve high performance and large capacity gain. This is achieved by making use of timing advance mechanisms; to ensure that the desired signals from the cooperating cells reach the mobile station at exactly the same time. The Joint Leakage Suppression (JLS) precoder is a suitable linear precoding scheme in this scenario. In this paper, JLS performance analysis is considered under real assumptions such as the presence of asynchronous reception of the interfering signals and the effect of the finite capacity backhaul links. The effect of quantizing the feedback channel on the sum rate is derived, and a tight upper bound of the rate loss due to quantization is obtained. Simulation results are provided to validate these results.
\end{abstract}

Keywords-CoMP transmission, JLS precoder, asynchronous interference, quantized feedback.

\section{INTRODUCTION}

Coordinated Multi-Point transmission/reception (CoMP) has been considered by the $3^{\text {rd }}$ Generation Partnership Project (3GPP) as a candidate technique for LTE-Advanced systems [1], [2]. CoMP has been proposed to increase the average cell throughput and the cell edge user throughput in both the uplink and downlink [3]. Several precoding schemes can be used to support CoMP transmission and achieve these objectives. Among these schemes is the Dirty Paper Coding (DPC) scheme [4], [5], which achieves the capacity of MUMIMO downlink channel, however, its complexity stands up against its practical implementation. Other non-linear precoding techniques including Tomlinson-Harashima precoding [6], or multi-user detection in Mobile Stations (MSs) [7] are approaching such capacity, but they still suffer from prohibitive complexity. Therefore, there is great interest in linear precoding designs. Such linear precoding schemes have shown adequate performance. For example, the Zero Forcing (ZF) precoder only loses about $1.26 \mathrm{~dB}$ compared to DPC when having 10 transmit antennas and 5 receive antennas [8].

Joint processing transmission requires sharing the data stream of the MS among all cooperative BSs, in addition to the Channel State Information (CSI) and the propagation delay information from each BS to each MS to allow timing advance mechanisms to be used. However, such feedback requires infinite backhaul capacity. Practically, the backhaul has limited rate, and therefore each BS should consider quantizing the shared information with other cooperating BSs. Typically, CSI

Manuscript received March 10, 2016; revised September 28, 2016.

Y. Fahmy is with Electronics and Communication department, Cairo University, Egypt, e-mail: yfahmy@cws-cufe.org. is quantized and shared first in joint processing transmission, then only a sub-stream of user data or a quantized version of the antenna signals is shared among the base stations, which allows partial interference cancellation [9]. In [10], the impact of quantized and delayed CSI on the average achievable rate is derived. Accurate approximations on the expected sum rate of CoMP systems with imperfect CSI are also given.

Advancing the signal from each BS in the cooperative set to reach the intended user at exactly the same time, results in asynchronous reception of these signals at other users. This opened the way for different precoding schemes that can account for the problem of asynchronous interference.

Taking the ZF precoder that can achieve the ergodic sum capacity in limit of large number of active users [11], it can no longer cancel all the interference in the presence of asynchronous interference, except under severe limitations, where the number of antennas of all users must be less than the number of transmit antennas in each BS [12]. In [13], a new MMSE design was investigates in CoMP system. The proposed scheme is robust to the asynchronous interference and the channel quantization errors. Joint Leakage Suppression (JLS) was proposed in [14] to minimize the co-channel interference (CCI) instead of canceling it out completely. This technique aims at maximizing the Signal to Leakage plus Noise Ratio (SLNR) for all users simultaneously. Where, compared to zero-forcing precoding, JLS method can minimize the asynchronous interference without imposing any conditions on the relation between the number of transmit and receive antennas. Moreover, it avoids noise enhancement.

In this paper, the finite rate feedback-JLS precoder is presented where a tight upper bound for the rate loss due to channel quantization is derived in two different scenarios; the synchronous interference scenario, and the more realistic scenario of asynchronous interference. The rate loss of other precoders has been studied in the literature for multi-cell systems. In [15] and [16], the rate loss, as a function of the quantization error when using generalized eigenvector beamforming in a rate-limited environment, is studied. However, the expression derived for the rate loss is kept as a function of the quantization error of both the desired and interfering channel vectors, while this quantization error has not been analyzed. This quantization error analysis differs in case of multi-cell MIMO than the case of single-cell MIMO, and that is what will be presented in the following section. Moreover, no analysis has been carried out for the JLS precoder before. As explained earlier, this precoder is a practical choice for the implementation of the multi-cell MIMO in the presence of asynchronous interference. 


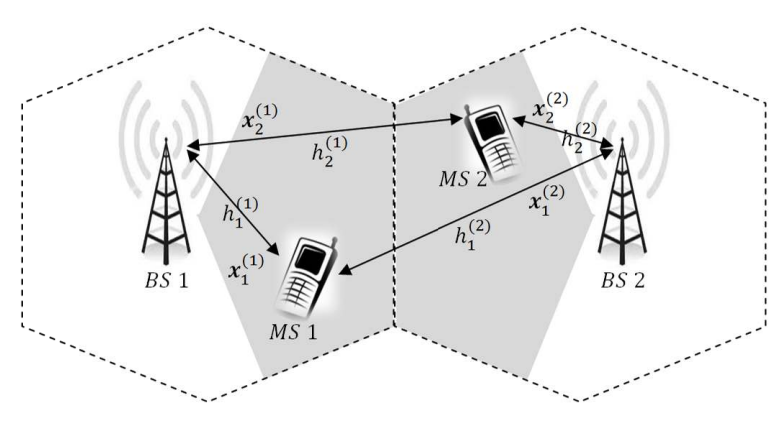

Fig. 1. System Model

The rest of this paper is organized as follows. Section II presents the asynchronous interference systems model, then the design criteria of the JLS precoder is given. In section III. the JLS rate is derived. Section IV presents the analysis of the quantization error of the random vector quantizer in the MUCoMP network, then the rate loss due to feedback quantization is presented, in the synchronous idealized scenario. The proof is then extended to the more realistic case of asynchronous interference. Section $\mathrm{V}$ presents the simulation results, verifying the analytical results obtained for the rate loss, under both scenarios. Finally, Section VI concludes the paper.

\section{ASYNCHRONOUS INTERFERENCE SySTEM MODEL}

The system model considers a MU-CoMP network [1] where $B$ Base Stations (BSs), having $N_{T}$ antennas each, cooperate together to send data to $K$ Mobile Stations (MSs), with $N_{R}$ antennas each. The cooperative BSs together transmit $L_{k}$ data streams to MS $k$, where the transmit vector from BS $b$ to MS $k$ is linearly precoded by the $N_{T} \times L_{k}$ matrix $\boldsymbol{T}_{k}^{(b)}$. If $\boldsymbol{s}_{k}(m)$ is the zero-mean data vector, of size $L_{k} \times 1$ at time $m$ meant for MS $k$, then the transmit vector from BS $b$ to MS $k$ is $\boldsymbol{x}_{k}^{(b)}(m)=\boldsymbol{T}_{k}^{(b)} \boldsymbol{s}_{k}(m)$. The data vector has normalized power such that $E\left[\boldsymbol{s}_{k}(m) \boldsymbol{s}_{k}(m)^{\dagger}\right]=\mathbf{I}_{L_{k}}$, where $\mathbf{I}_{L_{k}}$ is the identity matrix of size $L_{k}$ and [. $]^{\dagger}$ is the Hermitian transpose. Data vectors for different users are independent of each other, i.e., $E\left[\boldsymbol{s}_{k}(m) \boldsymbol{s}_{l}(m)^{\dagger}\right]=\mathbf{0}$, for $k \neq l$. A linear modulation with a unit energy baseband signature waveform $g(t)$ of duration $T_{s}$ is used. The system model is shown in Fig. 1.

Assume that $\boldsymbol{h}_{k}^{(b)}$ is the $N_{R} \times N_{T}$ baseband matrix representation of the channel from $\mathrm{BS} b$ to $\mathrm{MS} k$, having complex Gaussian elements. Different links are assumed to be independent of each other and undergo frequency-flat Rayleigh fading. Also, block fading channel model with large enough coherence time is used, such that the channel fading remains the same over the duration in which $\boldsymbol{T}_{k}^{(b)}$ is used.

In order to guarantee simultaneous reception of $\left\{\boldsymbol{x}_{k}^{(b)}(m)\right\}_{b=1}^{B}$ at MS $k$, each BS $b$ advances the transmission time of $\boldsymbol{x}_{k_{B}}^{(b)}(m)$ by $\Delta \tau_{k}^{(b)}=\tau_{k}^{(b)}-\tau_{k}^{\left(b_{k}\right)}$ such that all $\left\{\boldsymbol{x}_{k}^{(b)}(m)\right\}_{b=1}^{B}$ arrive with the same delay $\tau_{k}^{\left(b_{k}\right)}$, where $b_{k}$ is the closest $b \overline{\mathrm{BS}}$ to MS $k$. The equivalent received baseband signal at MS $k$ is

$$
\begin{array}{r}
\boldsymbol{r}_{k}(t)=\sum_{m=0}^{\infty}\left(g\left(t-m T s-\tau_{k}^{\left(b_{k}\right)}\right) \boldsymbol{H}_{k} \boldsymbol{x}_{k}(m)\right)+\boldsymbol{n}_{k}(t) \\
+\sum_{\substack{j=1 \\
j \neq k}}^{K} \sum_{b=1}^{B} \sum_{m=0}^{\infty}\left(g\left(t-m T s-\tau_{k}^{(b)}+\triangle \tau_{j}^{(b)}\right) \boldsymbol{h}_{k}^{(b)} \boldsymbol{x}_{j}^{(b)}(m)\right),
\end{array}
$$

where $\boldsymbol{n}_{k}(t)$ is the additive white Gaussian noise vector with zero mean and variance $N_{0} . \boldsymbol{H}_{k}$ is the $N_{R} \times B N_{T}$ composite channel matrix of user $k$ defined as $\boldsymbol{H}_{k}=\left[\boldsymbol{h}_{k}^{(1)}, \ldots, \boldsymbol{h}_{k}^{(B)}\right]$, and $\boldsymbol{x}_{k}(m)$ is the composite data matrix, defined as $\boldsymbol{x}_{k}(m)=$ $\left[\boldsymbol{x}_{k}^{(1)}(m)^{\dagger}, \ldots, \boldsymbol{x}_{k}^{(B)}(m)^{\dagger}\right]^{\dagger}$.

In the asynchronous interference case, when the received signal is passed through a filter matched to $g\left(t-m T s-\tau_{k}^{\left(b_{k}\right)}\right)$, the resulting signal is given by

$$
\begin{aligned}
\boldsymbol{y}_{k}(m) & =\boldsymbol{H}_{k} \boldsymbol{T}_{k} \boldsymbol{s}_{k}(m)+\boldsymbol{n}_{k}(m) \\
& +\sum_{\substack{j=1 \\
j \neq k}}^{K} \sum_{b=1}^{B} \boldsymbol{h}_{k}^{(b)} \boldsymbol{T}_{j}^{(b)}(m) \boldsymbol{i}_{j k}^{(b)}(m),
\end{aligned}
$$

where $\boldsymbol{i}_{j k}^{(b)}(m)$ is the asynchronous interference arising from two consecutive symbols with indices $m_{j k}^{(b)}$ and $m_{j k}^{(b)}+1$ at MS $k$ from the signal transmitted by BS $b$ for MS $j$.

The statistical characteristics of the asynchronous interference are $E\left[\boldsymbol{i}_{j k}^{(b)}(m)\right]=\mathbf{0}$ and

$$
E\left[\boldsymbol{i}_{j_{1} k}^{\left(b_{1}\right)}(m) \boldsymbol{i}_{j_{2} k}^{\left(b_{2}\right)}(m)^{\dagger}\right]= \begin{cases}\mathbf{0} & j_{1} \neq j_{2} \neq k \\ \beta_{j k}^{\left(b_{1}, b_{2}\right)} \boldsymbol{I}_{l_{j}} & j_{1}=j_{2}=j \neq k \\ \boldsymbol{I}_{l_{j}} & j_{1}=j_{2}=j=k\end{cases}
$$

where the asynchronous interference correlation $\beta_{j k}^{\left(b_{1}, b_{2}\right)}$ is given in [12].

\section{JLS PRECODER PERFORMANCE}

The goal of the JLS precoder [14] is to jointly optimize the transmitter precoding matrices $\boldsymbol{T}_{k}$ to maximize the SLNR, subject to the following two constraints: first, $\operatorname{Tr}\left\{\boldsymbol{T}_{k}^{\dagger} \boldsymbol{T}_{k}\right\} \leq P_{k}^{t x}$, where $P_{k}^{t x}$ is the transmitted power to MS $k$, for $1 \leq k \leq K$. This uniform per-MS power constraint, $P_{k}^{t x}=P_{T}$, for all $k$, ensures "power fairness" for different users. Even though, the per-BS power constraint makes more physical sense, the advantage of the MS-specific power constraint is that it is more amenable to analytically tractable solutions. The second design constraint we have is that $B . N_{T} \geq \sum_{k=1}^{K} L_{k}$.

The SLNR is defined as the ratio between the power of the desired signal at MS $k$ and the sum of noise power plus total interference power (leakage) due to MS $k$ at all other MSs. From which the SLNR, for the idealized synchronous case, was given in [14] by

$\mathrm{SLNR}_{k}=\frac{P_{k}^{t x} \cdot \operatorname{Tr}\left(\boldsymbol{Q}_{k}^{\dagger} \boldsymbol{H}_{k}^{\dagger} \boldsymbol{H}_{k} \boldsymbol{Q}_{k}\right)}{\operatorname{Tr}\left(\boldsymbol{Q}_{k}^{\dagger}\left(N_{0} N_{R} \boldsymbol{I}_{B N_{T}}+P_{k}^{t x} \tilde{\boldsymbol{H}}_{k}^{\dagger} \tilde{\boldsymbol{H}}_{k}\right) \boldsymbol{Q}_{k}\right)}$

where $\boldsymbol{Q}_{k}=\frac{1}{\sqrt{P_{k}^{t x}}} \boldsymbol{T}_{k}$ and 
$\tilde{\boldsymbol{H}}_{k}=\left[\boldsymbol{H}_{1} \boldsymbol{H}_{2} \cdots \boldsymbol{H}_{k-1} \boldsymbol{H}_{k+1} \cdots \boldsymbol{H}_{K}\right]$ represents all the channels except the channel of the intended user $k$.

While the SLNR was calculated in the case of asynchronous interference in [12] as

$$
\mathrm{SLNR}_{k}=\frac{P_{k}^{t x} \cdot \operatorname{Tr}\left(\boldsymbol{Q}_{k}^{\dagger} \boldsymbol{H}_{k}^{\dagger} \boldsymbol{H}_{k} \boldsymbol{Q}_{k}\right)}{\operatorname{Tr}\left(\boldsymbol{Q}_{k}^{\dagger}\left(N_{0} N_{R} \boldsymbol{I}_{B N_{T}}+P_{k}^{t x} \sum_{\substack{j=1 \\ j \neq k}}^{K} \boldsymbol{W}_{k j}\right) \boldsymbol{Q}_{k}\right)}
$$

where

$$
\boldsymbol{W}_{k j}=\left[\begin{array}{ccc}
\beta_{k j}^{(1,1)} \boldsymbol{h}_{j}^{(1)^{\dagger}} \boldsymbol{h}_{j}^{(1)} & \ldots & \beta_{k j}^{(1, B)} \boldsymbol{h}_{j}^{(1)^{\dagger}} \boldsymbol{h}_{j}^{(B)} \\
\beta_{k j}^{(2,1)} \boldsymbol{h}_{j}^{(2)^{\dagger}} \boldsymbol{h}_{j}^{(1)} & \ldots & \beta_{k j}^{(2, B)} \boldsymbol{h}_{j}^{(2)^{\dagger}} \boldsymbol{h}_{j}^{(B)} \\
\vdots & \ddots & \vdots \\
\beta_{k j}^{(B, 1)} \boldsymbol{h}_{j}^{(B)^{\dagger}} \boldsymbol{h}_{j}^{(1)} & \ldots & \beta_{k j}^{(B, B)} \boldsymbol{h}_{j}^{(B)^{\dagger}} \boldsymbol{h}_{j}^{(B)}
\end{array}\right]
$$

Let

$$
\boldsymbol{Y}_{k}=\frac{P_{k}^{t x}}{N_{0}} \boldsymbol{H}_{k}^{\dagger} \boldsymbol{H}_{k}
$$

and

$$
\boldsymbol{Z}_{k}=N_{R} \boldsymbol{I}_{B N_{T}}+\frac{P_{k}^{t x}}{N_{0}} \sum_{\substack{j=1 \\ j \neq k}}^{K} \boldsymbol{W}_{k j}
$$

then (3) is simplified to

$$
\mathrm{SLNR}_{k}=\frac{\operatorname{Tr}\left(\boldsymbol{Q}_{k}^{\dagger} \boldsymbol{Y}_{k} \boldsymbol{Q}_{k}\right)}{\operatorname{Tr}\left(\boldsymbol{Q}_{k}^{\dagger} \boldsymbol{Z}_{k} \boldsymbol{Q}_{k}\right)}
$$

In order to get more insights into the behavior of the JLS precoder, we will consider the practical case of having one receiving antenna at the MSs $\left(N_{R}=1\right.$ and consequently $L_{k}=$ $1)$. This case is also mathematically tractable and will result in a closed form solution of the rate loss.

\section{A. Maximum SLNR}

Assume the case of $L_{k}=1, \boldsymbol{Q}_{k}$ is a vector of size $N_{T} \times 1$ and since $\boldsymbol{Y}_{k}$ is Hermitian and $\boldsymbol{Z}_{k}$ is positive definite with real diagonal elements, equation (4) can be further simplified to take the form of a generalized Rayleigh quotient [17], where $\mathrm{SLNR}_{k}=\frac{\boldsymbol{Q}_{k}^{\dagger} \boldsymbol{Y}_{k} \boldsymbol{Q}_{k}}{\boldsymbol{Q}_{k}^{\dagger} \boldsymbol{Z}_{k} \boldsymbol{Q}_{k}}$.

Define $C$ as the Cholesky decomposition of the matrix $\boldsymbol{Z}_{k}=\boldsymbol{C}^{\dagger} \boldsymbol{C}$ and $\boldsymbol{D}=\boldsymbol{C}^{\dagger^{-1}} \boldsymbol{Y}_{k} \boldsymbol{C}^{-1}$, the SLNR can be simplified to the Rayleigh Quotient $R\left(\boldsymbol{D}, \boldsymbol{C} \boldsymbol{Q}_{k}\right)=\frac{\left(\boldsymbol{C} \boldsymbol{Q}_{\boldsymbol{k}}\right)^{\dagger} \boldsymbol{D}\left(\boldsymbol{C} \boldsymbol{Q}_{\boldsymbol{k}}\right)}{\left(\boldsymbol{C} \boldsymbol{Q}_{\boldsymbol{k}}\right)^{\dagger}\left(\boldsymbol{C \boldsymbol { Q } _ { k }}\right)}$, where $\left|\boldsymbol{C} \boldsymbol{Q}_{k}\right|=1$. This Rayleigh quotient reaches its maximum value of $\lambda_{\max }$ (the maximum eigenvalue of the matrix $D$ ) when $C Q_{k}=\boldsymbol{v}_{\max }$, which is the corresponding eigenvector of the matrix $D$.

Starting by $\boldsymbol{D} \boldsymbol{C} \boldsymbol{Q}_{k}=\lambda_{\max } \boldsymbol{C} \boldsymbol{Q}_{k}$, we get $\boldsymbol{Z}_{k}^{-1} \boldsymbol{Y}_{k} \boldsymbol{Q}_{k}=$ $\lambda_{\max } \boldsymbol{Q}_{k}$, then $\boldsymbol{Q}_{k}$ is the eigenvector of the matrix $\boldsymbol{Z}_{k}^{-1} \boldsymbol{Y}_{k}$ corresponding to its largest eigenvalue.

The maximum SLNR can then be given at this $\boldsymbol{Q}_{k}$ as

$$
\begin{array}{r}
\max \left(\mathrm{SLNR}_{k}\right)=\max \left(R\left(\boldsymbol{D}, \boldsymbol{C} \boldsymbol{Q}_{k}\right)\right) \\
=\left(\boldsymbol{C} \boldsymbol{Q}_{\boldsymbol{k}}\right)^{\dagger} \boldsymbol{D}\left(\boldsymbol{C} \boldsymbol{Q}_{\boldsymbol{k}}\right)=\boldsymbol{Q}_{k}^{\dagger} \boldsymbol{Y}_{k} \boldsymbol{Q}_{k}=\lambda_{\max }
\end{array}
$$

It is worth noting that at this maximum condition $\left|\boldsymbol{H}_{k} \boldsymbol{Q}_{k}\right|^{2}=\frac{N_{0}}{P_{k}^{t x}} \lambda_{\max }$ and $\boldsymbol{Q}_{k}^{\dagger} \boldsymbol{Z}_{k} \boldsymbol{Q}_{k}=1$ then

$$
\boldsymbol{Q}_{k}^{\dagger}\left(N_{R} \boldsymbol{I}_{B N_{T}}+\frac{P_{k}^{t x}}{N_{0}} \sum_{\substack{j=1 \\ j \neq k}}^{K} \boldsymbol{W}_{k j}\right) \boldsymbol{Q}_{k}=1
$$

at $N_{R}=1$

$$
\boldsymbol{Q}_{k}^{\dagger} \boldsymbol{Q}_{k}+\frac{P_{k}^{t x}}{N_{0}} \boldsymbol{Q}_{k}^{\dagger} \sum_{\substack{j=1 \\ j \neq k}}^{K} \boldsymbol{W}_{k j} \boldsymbol{Q}_{k}=1
$$

from the orthonormality condition $\boldsymbol{Q}_{k}^{\dagger} \boldsymbol{Q}_{k}=1$, then

$$
\boldsymbol{Q}_{k}^{\dagger} \sum_{\substack{j=1 \\ j \neq k}}^{K} \boldsymbol{W}_{k j} \boldsymbol{Q}_{k}=0
$$

Using the definition of $\boldsymbol{W}_{k j}$, this equation can be expanded to

$$
\sum_{\substack{j=1 \\ j \neq k}}^{K} \sum_{b_{i}=1}^{B} \sum_{b_{l}=1}^{B} \beta_{j k}^{\left(b_{i}, b_{l}\right)} \boldsymbol{Q}_{k}^{\left(b_{l}\right) \dagger} \boldsymbol{h}_{j}^{\left(b_{l}\right) \dagger} \boldsymbol{h}_{j}^{\left(b_{i}\right)} \boldsymbol{Q}_{k}^{\left(b_{i}\right)}=0
$$

Given the quantized channel $\hat{\boldsymbol{H}}_{j}$, the precoding vector $\boldsymbol{Q}_{k}$ is calculated to satisfy

$$
\sum_{\substack{j=1 \\ j \neq k}}^{K} \sum_{b_{i}=1}^{B} \sum_{b_{l}=1}^{B} \beta_{j k}^{\left(b_{i}, b_{l}\right)} \boldsymbol{Q}_{k}^{\left(b_{l}\right) \dagger} \hat{\boldsymbol{h}}_{j}^{\left(b_{l}\right) \dagger} \hat{\boldsymbol{h}}_{j}^{\left(b_{i}\right)} \boldsymbol{Q}_{k}^{\left(b_{i}\right)}=0
$$

as a design criterion.

\section{B. Maximum achievable Rate}

The JLS rate is given as a function of $P$, the SNR per MS, in [12] as

$$
\begin{gathered}
R_{J L S}(P)= \\
K E_{H}\left[\log _{2}\left|\boldsymbol{I}_{N_{R}}+\boldsymbol{\phi}_{k, J L S}^{-1} \boldsymbol{H}_{k} \boldsymbol{T}_{k, J L S} \boldsymbol{T}_{k, J L S}^{\dagger} \boldsymbol{H}_{k}^{\dagger}\right|\right]
\end{gathered}
$$

where the expectation $E_{H}$ [.] is held over the channel $H$ and $\phi_{k}$ is the covariance of noise plus interference for MS $k$. It is given by

$$
\phi_{k, J L S}=
$$

$$
\sum_{\substack{j=1 \\ j \neq k}}^{K} \sum_{b_{i}=1}^{B} \sum_{b_{l}=1}^{B} \beta_{j k}^{\left(b_{i}, b_{l}\right)} \boldsymbol{h}_{k}^{\left(b_{i}\right)} \boldsymbol{T}_{j, J L S}^{\left(b_{i}\right)} \boldsymbol{T}_{j, J L S}^{\left(b_{l}\right) \dagger} \boldsymbol{h}_{k}^{\left(b_{l}\right) \dagger}+N_{0} \boldsymbol{I}_{N_{R}}
$$

For $N_{R}=1$ and given that $\boldsymbol{H}_{k} \boldsymbol{T}_{k} \boldsymbol{T}_{k}^{\dagger} \boldsymbol{H}_{k}^{\dagger}=\left|\boldsymbol{H}_{k} \boldsymbol{T}_{k}\right|^{2}=$ $P_{k}^{t x}\left|\boldsymbol{H}_{k} \boldsymbol{Q}_{k, J L S}\right|^{2}$ and $P=\frac{P_{k}^{t x}}{N_{0}}, R_{J L S}(P)$ reduces to

$$
R_{J L S}(P)=K E_{H}\left[\log _{2}\left(1+\frac{P\left|\boldsymbol{H}_{k} \boldsymbol{Q}_{k, J L S}\right|^{2}}{1+P \cdot \gamma_{k, J L S}}\right)\right]
$$


where

$$
\gamma_{k, J L S}=\sum_{\substack{j=1 \\ j \neq k}}^{K} \sum_{b_{i}=1}^{B} \sum_{b_{l}=1}^{B} \beta_{j k}^{\left(b_{i}, b_{l}\right)} \boldsymbol{h}_{k}^{\left(b_{i}\right)} \boldsymbol{Q}_{j, J L S}^{\left(b_{i}\right)} \boldsymbol{Q}_{j, J L S}^{\left(b_{l}\right) \dagger} \boldsymbol{h}_{k}^{\left(b_{l}\right) \dagger}
$$

For perfect channel knowledge, $\gamma_{k, J L S}=0$ from (7), then

$$
R_{J L S}(P)=K E_{H}\left[\log _{2}\left(1+P\left|\boldsymbol{H}_{k} \boldsymbol{Q}_{k, J L S}\right|^{2}\right)\right]
$$

\section{QUANTIZED FEEDBACK JLS PRECODER}

In the available literature, scalar quantization, vector quantization (VQ) and matrix quantization have all been used to quantize CSI. It is now well established in the single data stream case that projecting the MIMO channel to an appropriate vector channel yields better performance than full channel scalar quantization with the same feedback overhead [18]. In the case of the single data stream case, the MIMO channel is projected to a vector channel. This was proved to yield better performance than full channel scalar quantization providing the same feedback overhead [18]. This has led to considerable research in VQ, which reduces the feedback overhead by allocating bits in the proper vector direction. For the case of having multiple data streams, the channel matrix is projected to the appropriate channel vector using one of the combining schemes proposed in the literature, for example eigenbased combining [19], quantization based combining [20], and maximum expected signal combining [21]. In the multi user case, we can apply the same principle of VQ to each user channel individually. The vector quantization is performed using a quantization codebook that is known at the BSs. And since the optimal vector quantizer for this problem is not known in general, Random Vector Quantizer (RVQ) is used [22]. Quantization schemes require a measure of the distance between the two vectors, where two commonly used measures are the chordal and euclidean distances. Since the former ensures a higher inner product between the original and quantized channel, in the limited feedback scenario [23], it is the one used in this paper.

\section{A. Random Vector Quantization Error Analysis}

In VQ, $n$ feedback bits are sent as the channel index to the master cell or other cooperating BSs. A quantization codebook C consisting of $2^{n} N_{T}$-dimensional unit norm vectors $\mathbf{C}=\left\{w_{1}, \ldots, w_{2^{n}}\right\}$ is used. Each of the codewords is independently drawn from the isotropic distribution on the $N_{T}$-dimensional unit sphere. We analyze the performance by averaging over the distribution of all choices of such codebooks.

Defining $d$ as the chordal distance between the channel vector and its quantized version [24] allows it to be used as the magnitude of quantization error. The average value of the quantization error of the composite channel vector $\boldsymbol{H}_{k}$, of size $1 \times B N_{T}$, can be proved from the quantization errors of its consisting channel vectors $\boldsymbol{h}_{k}^{(b)}$ for $b=1, \ldots, B$ as

$$
\begin{aligned}
d & =\sin ^{2}\left(\angle\left(\boldsymbol{H}_{\boldsymbol{k}}, \hat{\boldsymbol{H}}_{\boldsymbol{k}}\right)\right)=1-\left(\frac{\left|\boldsymbol{H}_{\boldsymbol{k}} \hat{\boldsymbol{H}}_{\boldsymbol{k}}^{\dagger}\right|^{2}}{\left\|\boldsymbol{H}_{\boldsymbol{k}}\right\|^{2} \cdot\left\|\hat{\boldsymbol{H}}_{\boldsymbol{k}}\right\|^{2}}\right) \\
& =1-\left(\frac{\left|\sum_{b=1}^{B} \boldsymbol{h}_{k}^{(b)} \hat{\boldsymbol{h}}_{k}^{(b) \dagger}\right|^{2}}{\left\|\boldsymbol{H}_{k}\right\|^{4}}\right)
\end{aligned}
$$

since both the channel and the quantized vectors have the same gain, and

$$
\cos ^{2}\left(\angle\left(\boldsymbol{H}_{\boldsymbol{k}}, \hat{\boldsymbol{H}}_{\boldsymbol{k}}\right)\right)=\left(\frac{\left|\sum_{b=1}^{B} \boldsymbol{h}_{k}^{(b)} \hat{\boldsymbol{h}}_{k}^{(b) \dagger}\right|^{2}}{\left\|\boldsymbol{H}_{k}\right\|^{4}}\right)
$$

By defining $a_{b}=\cos \left(\angle\left(\boldsymbol{h}_{k}^{(b)}, \hat{\boldsymbol{h}}_{k}^{(b)}\right)\right)$, we can define the quantity $\alpha$ as follows:

$$
\begin{aligned}
\alpha & =\left\|\boldsymbol{H}_{k}\right\|^{4} \cdot \cos ^{2}\left(\angle\left(\boldsymbol{H}_{\boldsymbol{k}}, \hat{\boldsymbol{H}}_{\boldsymbol{k}}\right)\right) \\
& =\sum_{j=1}^{B} \sum_{l=1}^{B} a_{b_{j}} a_{b_{l}}\left\|\hat{\boldsymbol{h}}_{k}^{\left(b_{j}\right)}\right\|^{2}\left\|\hat{\boldsymbol{h}}_{k}^{\left(b_{l}\right)}\right\|^{2}
\end{aligned}
$$

And by taking the expectation of both sides, we get

$$
\begin{aligned}
E(\alpha) & =E\left(\left\|\boldsymbol{H}_{k}\right\|^{4}\right) \cdot E\left(\cos ^{2}\left(\angle\left(\boldsymbol{H}_{\boldsymbol{k}}, \hat{\boldsymbol{H}}_{\boldsymbol{k}}\right)\right)\right) \\
& =E\left(\sum_{j=1}^{B} \sum_{l=1}^{B} a_{b_{j}} a_{b_{l}}\left\|\hat{\boldsymbol{h}}_{k}^{\left(b_{j}\right)}\right\|^{2}\left\|\hat{\boldsymbol{h}}_{k}^{\left(b_{l}\right)}\right\|^{2}\right)
\end{aligned}
$$

where we used here the fact that the angle between two vectors is independent of the values of their norm. Finally,

$$
\begin{gathered}
E(\alpha)=\sum_{i=1}^{B} E\left(a_{b_{i}}^{2}\right) \cdot E\left(\left\|\hat{\boldsymbol{h}}_{k}^{\left(b_{i}\right)}\right\|^{4}\right) \\
+2 \sum_{j=1}^{B} \sum_{l=j+1}^{B} E\left(a_{b_{j}}\right) E\left(a_{b_{l}}\right) E\left(\left\|\hat{\boldsymbol{h}}_{k}^{\left(b_{j}\right)}\right\|^{2}\right) E\left(\left\|\hat{\boldsymbol{h}}_{k}^{\left(b_{l}\right)}\right\|^{2}\right) .
\end{gathered}
$$

In [25] the error performance of the RVQ scheme has been analyzed in the case of point to point MISO systems, and the expected quantization error has been bounded by the following bounds

$$
\left(\frac{N_{T}-1}{N_{T}}\right) 2^{\frac{-n}{N_{T}-1}}<E\left[\sin ^{2}\left(\angle\left(\boldsymbol{H}_{\boldsymbol{k}}, \hat{\boldsymbol{H}}_{\boldsymbol{k}}\right)\right)\right]<2^{\frac{-n}{N_{T}-1}}
$$

therefore the lower bound of each of the terms $E\left(a_{b_{j}}^{2}\right)$ is given by

$$
E\left(a_{b_{j}}^{2}\right)=E\left(\cos ^{2}\left(\angle\left(\boldsymbol{h}_{k}^{\left(b_{j}\right)}, \hat{\boldsymbol{h}}_{k}^{\left(b_{j}\right)}\right)\right)\right) \geq 1-2^{\frac{-n}{N_{T}-1}},
$$

while $E\left(a_{b_{j}}\right)=0$ as the codebook vectors are isotropically distributed in the $2^{n}$ dimensional space, then the channel vector can be mapped to a certain vector or the one in the opposite direction with the same probability.

To get $E\left(\left\|\hat{\boldsymbol{h}}_{k}^{\left(b_{i}\right)}\right\|^{2}\right)$, we use the fact that the squared norm of a vector consisting of $r$ Gaussian i.i.d. random variables, each with zero mean and variance $\sigma^{2}$, is a chisquared distribution with $r$ degrees of freedom. The norm of each channel vector, as well as the quantized one, is the summation of two independent chi-squared random variables, one for the real part of the vector and one for the imaginary 
part. Each part has $N_{T}$ degrees of freedom, and variance $\frac{L_{k}^{\left(b_{i}\right)}}{2}$ where $L_{k}^{\left(b_{i}\right)}$ is the path loss of the channel between BS $b_{(i)}$ and MS $k$.

Then

$$
\begin{aligned}
E\left(\left\|\hat{\boldsymbol{h}}_{k}^{\left(b_{i}\right)}\right\|^{2}\right)= & \frac{L_{k}^{\left(b_{i}\right)}}{2} N_{T}+\frac{L_{k}^{\left(b_{i}\right)}}{2} N_{T}=L_{k}^{\left(b_{i}\right)} N_{T} \\
\operatorname{Var}\left(\left\|\hat{\boldsymbol{h}}_{k}^{\left(b_{i}\right)}\right\|^{2}\right) & =2 N_{T}\left(\frac{L_{k}^{\left(b_{i}\right)}}{2}\right)^{2}+2 N_{T}\left(\frac{L_{k}^{\left(b_{i}\right)}}{2}\right)^{2} \\
& =N_{T}\left(L_{k}^{\left(b_{i}\right)}\right)^{2}
\end{aligned}
$$

and hence

$$
\begin{aligned}
E\left(\left\|\hat{\boldsymbol{h}}_{k}^{\left(b_{i}\right)}\right\|^{4}\right) & =\operatorname{Var}\left(\left\|\hat{\boldsymbol{h}}_{k}^{\left(b_{i}\right)}\right\|^{2}\right)+E^{2}\left(\left\|\boldsymbol{h}_{k}^{\left(b_{i}\right)}\right\|^{2}\right) \\
& =\left(L_{k}^{\left(b_{i}\right)}\right)^{2}\left(N_{T}+N_{T}^{2}\right) .
\end{aligned}
$$

Then substituting in (14), we find that

$$
E(\alpha) \geq\left(N_{T}+N_{T}^{2}\right)\left(1-2^{\frac{-n}{N_{T}-1}}\right) \sum_{i=1}^{B}\left(L_{k}^{\left(b_{i}\right)}\right)^{2} .
$$

Since $\left\|\hat{\boldsymbol{H}}_{k}\right\|^{2}=\sum_{i=1}^{B}\left\|\hat{\boldsymbol{h}}_{k}^{\left(b_{i}\right)}\right\|^{2}$, then the norm $\left\|\hat{\boldsymbol{H}}_{k}\right\|^{2}$ has a mean value of $E\left(\left\|\hat{\boldsymbol{H}}_{k}\right\|^{2}\right)=N_{T} \cdot \sum_{i=1}^{B} L_{k}^{\left(b_{i}\right)}$, and variance given by $\operatorname{Var}\left(\left\|\hat{\boldsymbol{H}}_{k}^{\left(b_{i}\right)}\right\|^{2}\right)=N_{T} \cdot \sum_{i=1}^{B}\left(L_{k}^{\left(b_{i}\right)}\right)^{2}$, then

$$
E\left(\left\|\hat{\boldsymbol{H}}_{k}\right\|^{4}\right)=N_{T} \cdot \sum_{i=1}^{B}\left(L_{k}^{\left(b_{i}\right)}\right)^{2}+\left(N_{T} \cdot \sum_{i=1}^{B} L_{k}^{\left(b_{i}\right)}\right)^{2}
$$

By substituting with this value and the value of $E(\alpha)$ in 13 , the value of $E\left(\cos ^{2}\left(\angle\left(\boldsymbol{H}_{\boldsymbol{k}}, \hat{\boldsymbol{H}}_{\boldsymbol{k}}\right)\right)\right)$ is bounded by

$$
\begin{gathered}
E\left(\cos ^{2}\left(\angle\left(\boldsymbol{H}_{\boldsymbol{k}}, \hat{\boldsymbol{H}}_{\boldsymbol{k}}\right)\right)\right) \geq \\
\frac{\left(N_{T}+N_{T}^{2}\right)\left(1-2^{\frac{-n}{N_{T}-1}}\right) \sum_{i=1}^{B}\left(L_{k}^{\left(b_{i}\right)}\right)^{2}}{N_{T} \cdot \sum_{i=1}^{B}\left(L_{k}^{\left(b_{i}\right)}\right)^{2}+N_{T}^{2} \cdot\left(\sum_{i=1}^{B} L_{k}^{\left(b_{i}\right)}\right)^{2}}
\end{gathered}
$$

which can be reduced to the following form, in the case of having equal path losses,

$$
E\left(\cos ^{2}\left(\angle\left(\boldsymbol{H}_{\boldsymbol{k}}, \hat{\boldsymbol{H}}_{\boldsymbol{k}}\right)\right)\right) \geq \frac{B\left(N_{T}+N_{T}^{2}\right)\left(1-2^{\frac{-n}{N_{T}-1}}\right)}{B N_{T}+B^{2} N_{T}^{2}} .
$$

Therefore the mean value of the distance $d$ is bounded by

$$
\begin{aligned}
& E(d)=E\left(\sin ^{2}\left(\angle\left(\boldsymbol{H}_{\boldsymbol{k}}, \hat{\boldsymbol{H}}_{\boldsymbol{k}}\right)\right)\right) \\
& \leq 1-\frac{B\left(N_{T}+N_{T}^{2}\right)\left(1-2^{\frac{-n}{N_{T}-1}}\right)}{B N_{T}+B^{2} N_{T}^{2}} \\
& \leq 1-\frac{\left(1+N_{T}\right)}{\left(1+B N_{T}\right)}\left(1-2^{\frac{-n}{N_{T}-1}}\right)
\end{aligned}
$$

This is the upper bound of the quantization error resulting from random vector quantization when using chordal distance as the decision metric. This bound is used to find the rate loss in the next subsection.

\section{B. Rate Loss due to Quantization}

To quantify the performance degradation when the feedback channel is quantized in case of the JLS precoder, we define the rate gap $\triangle R(P)$ to be the difference between the per mobile throughput achieved by perfect CSIT-based JLS and finite-rate feedback-based JLS as

$$
\triangle R(P)=\frac{1}{K}\left[R_{J L S}(P)-R_{F B}(P)\right]
$$

The JLS rate, $R_{J L S}(P)$, is given by equation 10 , while the quantized feedback rate of the JLS precoder can be given by

$$
R_{F B}(P)=K E_{H, w}\left[\log _{2}\left(1+\frac{P\left|\boldsymbol{H}_{k} \boldsymbol{Q}_{k, F B}\right|^{2}}{1+P \cdot \gamma_{k, F B}}\right)\right]
$$

where the expectation $E_{H, w}[$.$] is held over the channel H$ and the quantization codebook vectors $w$, and

$$
\gamma_{k, F B}=\sum_{\substack{j=1 \\ j \neq k}}^{K} \sum_{b_{i}=1}^{B} \sum_{b_{l}=1}^{B} \beta_{j k}^{\left(b_{i}, b_{l}\right)} \boldsymbol{h}_{k}^{\left(b_{i}\right)} \boldsymbol{Q}_{j, F B}^{\left(b_{i}\right)} \boldsymbol{Q}_{j, F B}^{\left(b_{l}\right) \dagger} \boldsymbol{h}_{k}^{\left(b_{l}\right) \dagger}
$$

Then,

$$
\begin{aligned}
\triangle R(P) & =E_{H}\left[\log _{2}\left(1+P\left|\boldsymbol{H}_{k} \boldsymbol{Q}_{k, J L S}\right|^{2}\right)\right] \\
& -E_{H, w}\left[\log _{2}\left(1+\frac{P\left|\boldsymbol{H}_{k} \boldsymbol{Q}_{k, F B}\right|^{2}}{1+P \cdot \gamma_{k, F B}}\right)\right]
\end{aligned}
$$

Due to the use of the precoder, which tries to enhance the signal power and minimize the interference terms, the interference term $P \cdot \gamma_{k, F B}$ is much less than the received signal power $P\left|\boldsymbol{H}_{k} \boldsymbol{Q}_{k, F B}\right|^{2}$. The rate gap, $\triangle R(P)$, can then be bounded as

$$
\begin{aligned}
\triangle R(P) \leq & E_{H}\left[\log _{2}\left(1+P\left|\boldsymbol{H}_{k} \boldsymbol{Q}_{k, J L S}\right|^{2}\right)\right] \\
& -E_{H, w}\left[\log _{2}\left(1+P\left|\boldsymbol{H}_{k} \boldsymbol{Q}_{k, F B}\right|^{2}\right)\right] \\
& +E_{H, w}\left[\log _{2}\left(1+P \cdot \gamma_{k, F B}\right)\right] .
\end{aligned}
$$

This bound is valid taking into consideration the fact that $\gamma_{k, F B}$ is positive real and $\log (\cdot)$ is an increasing function.

Recall that the distribution of an isotropically random vector (which is the Rayleigh fading channel in our case) is not affected by multiplication with a unitary matrix [26]. It is the mathematical way to capture the notion that the vector is equally likely to point in any direction in the $N_{T}$ dimensional vector space. Using this result, $E_{H}\left[\log _{2}(1+\right.$ $\left.\left.P\left|\boldsymbol{H}_{k} \boldsymbol{Q}_{k, J L S}\right|^{2}\right)\right]=E_{H, w}\left[\log _{2}\left(1+P\left|\boldsymbol{H}_{k} \boldsymbol{Q}_{k, F B}\right|^{2}\right)\right]$. Then, $\triangle R(P) \leq E_{H, w}\left[\log _{2}\left(1+P \cdot \gamma_{k, F B}\right)\right]$. Using Jensen's inequality [27] the rate loss becomes bounded by

$$
\triangle R(P) \leq \log _{2}\left(1+E_{H, w}\left[P \cdot \gamma_{k, F B}\right]\right)
$$


1) Synchronous Interference Rate Loss: In case of having synchronous interference, $\beta_{j k}^{\left(b_{i}, b_{l}\right)}=1 \forall b_{i}, b_{l}$, then

$$
\begin{aligned}
\gamma_{k, F B} & =\sum_{\substack{j=1 \\
j \neq k}}^{K} \sum_{b_{i}=1}^{B} \sum_{b_{l}=1}^{B} \boldsymbol{h}_{k}^{\left(b_{i}\right)} \boldsymbol{Q}_{j, F B}^{\left(b_{i}\right)} \boldsymbol{Q}_{j, F B}^{\left(b_{l}\right) \dagger} \boldsymbol{h}_{k}^{\left(b_{l}\right) \dagger} \\
& =\sum_{\substack{j=1 \\
j \neq k}}^{K}\left|\boldsymbol{H}_{k} \boldsymbol{Q}_{j, F B}\right|^{2}
\end{aligned}
$$

and inequality 24 then becomes

$$
\triangle R(P) \leq \log _{2}\left(1+E_{H, w}\left[P \sum_{\substack{j=1 \\ j \neq k}}^{K}\left|\boldsymbol{H}_{k} \boldsymbol{Q}_{j, F B}\right|^{2}\right]\right)
$$

which is reduced to the following form, since all the mobile users' channels are independently quantized,

$$
\triangle R(P) \leq \log _{2}\left(1+(K-1) \cdot P \cdot E_{H, w}\left(\left|\boldsymbol{H}_{k} \boldsymbol{Q}_{j, F B}\right|^{2}\right)\right)
$$

And since $E\left(\left\|\boldsymbol{H}_{k}\right\|^{2}\right)=N_{T} \cdot\left(L_{k}^{(1)}+\cdots+L_{k}^{(B)}\right)$ where $L_{k}^{(b)}$ is the path loss coefficient of the channel between BS $b$ and MS $k$, then

$$
\begin{gathered}
\triangle R(P) \leq \\
\log _{2}\left(1+(K-1) P \sum_{b=1}^{B} L_{k}^{(b)} N_{T} E_{H, w}\left(\left|\overline{\boldsymbol{H}}_{k} \boldsymbol{Q}_{j, F B}\right|^{2}\right)\right)
\end{gathered}
$$

where $\overline{\boldsymbol{H}}_{k}=\frac{\boldsymbol{H}_{k}}{\left\|\boldsymbol{H}_{\boldsymbol{k}}\right\|}$ is the normalized composite channel vector.

Using RVQ to quantize the channel of each BS $\boldsymbol{h}_{k}^{(b)}$, then MS $k$ has a composite quantized channel vector of $\hat{\boldsymbol{H}}_{k}=$ $\left[\hat{\boldsymbol{h}}_{k}^{(1)}, \ldots, \hat{\boldsymbol{h}}_{k}^{(B)}\right] . \overline{\boldsymbol{H}}_{k}$ can be considered as the sum of two vectors, one in the direction of the quantized vector, and the other is isotropically distributed in the null space. Then

$$
\overline{\boldsymbol{H}}_{k}=\sqrt{1-d} \hat{\boldsymbol{H}}_{k}+\sqrt{d} \boldsymbol{S},
$$

where $d=\sin ^{2}(\theta)=1-\left|\overline{\boldsymbol{H}}_{k} \hat{\boldsymbol{H}}_{k}^{\dagger}\right|^{2}$ is the magnitude of the quantization error and $\boldsymbol{S}$ is a unit norm vector isotropically distributed in the null space of $\hat{\boldsymbol{H}}_{k}$.

The product of $\overline{\boldsymbol{H}}_{k}$ and $\boldsymbol{Q}_{j, F B}$ is then given by

$$
\begin{aligned}
\left|\overline{\boldsymbol{H}}_{k} \boldsymbol{Q}_{j, F B}\right|^{2} & =(1-d)\left|\hat{\boldsymbol{H}}_{k} \boldsymbol{Q}_{j, F B}\right|^{2}+(d)\left|\boldsymbol{S} \boldsymbol{Q}_{j, F B}\right|^{2} \\
& =d\left|\boldsymbol{S} \boldsymbol{Q}_{j, F B}\right|^{2}
\end{aligned}
$$

In case of having synchronous interference, (7) is reduced to

$$
\sum_{\substack{j=1 \\ j \neq k}}^{K}\left|\hat{\boldsymbol{H}}_{k} \boldsymbol{Q}_{j, F B}\right|^{2}=0
$$

therefore, the vector $\boldsymbol{Q}_{j, F B}$ is isotropically distributed in the $B N_{T}-1$ dimensional null space of $\hat{\boldsymbol{H}}_{k} \forall k \neq j$. And since $\boldsymbol{S}$ is also i.i.d. isotropic vector in the $B N_{T}-1$ dimensional null space of $\hat{\boldsymbol{H}}_{k}$, therefore the term $\left|\boldsymbol{S} \boldsymbol{Q}_{j, F B}\right|^{2}$ is beta distributed as $\beta\left(1, B N_{T}-2\right)$ random variable [28], having a mean value of $\frac{1}{B N_{T}-1}$. Accordingly, the mean value of $\left|\overline{\boldsymbol{H}}_{k} \boldsymbol{Q}_{j, F B}\right|^{2}$ is given by

$$
\begin{aligned}
E_{H, w}\left(\left|\overline{\boldsymbol{H}}_{k} \boldsymbol{Q}_{j, F B}\right|^{2}\right) & =E\left(d\left|\boldsymbol{S} \boldsymbol{Q}_{j, F B}\right|^{2}\right) \\
& =E(d) \cdot E\left(\left|\boldsymbol{S} \boldsymbol{Q}_{j, F B}\right|^{2}\right) \\
& =E\left(\sin ^{2}(\theta)\right) \cdot \frac{1}{B N_{T}-1}
\end{aligned}
$$

where the expectation of the quantization error has been proved in (19). By substituting these results in 26, we can find that the rate loss is upper bounded by the following bound

$$
\begin{array}{r}
\triangle R(P) \leq \log _{2}\left(1+(K-1) P \frac{\sum_{b=1}^{B} L_{k}^{(b)} N_{T}}{B N_{T}-1}\right. \\
\left.\cdot\left(1-\frac{\left(1+N_{T}\right)}{\left(1+B N_{T}\right)}\left(1-2^{\frac{-n}{N_{T}-1}}\right)\right)\right)
\end{array}
$$

2) Asynchronous Interference Rate Loss: To simplify the analysis and without loss of generality, we consider the case of having 2 BSs to get the rate loss of the JLS precoder in case of the presence of the more realistic scenario of Asynchronous interference. We have

$$
\gamma_{k, F B}=\sum_{\substack{j=1 \\ j \neq k}}^{K} \sum_{b_{i}=1}^{2} \sum_{b_{l}=1}^{2} \beta_{j k}^{\left(b_{i}, b_{l}\right)} \boldsymbol{h}_{k}^{\left(b_{i}\right)} \boldsymbol{Q}_{j, F B}^{\left(b_{i}\right)} \boldsymbol{Q}_{j, F B}^{\left(b_{l}\right) \dagger} \boldsymbol{h}_{k}^{\left(b_{l}\right) \dagger}
$$

since $\beta_{j k}^{\left(b_{i}, b_{l}\right)}=\beta_{j k}^{\left(b_{l}, b_{i}\right)}$, then

$$
\begin{aligned}
\gamma_{k, F B} & =\sum_{\substack{j=1 \\
j \neq k}}^{K}\left(\beta_{j k}^{\left(b_{i}, b_{i}\right)}\left|\boldsymbol{h}_{k}^{\left(b_{i}\right)} \boldsymbol{Q}_{j, F B}^{\left(b_{i}\right)}\right|^{2}\right. \\
& +\beta_{j k}^{\left(b_{l}, b_{l}\right)}\left|\boldsymbol{h}_{k}^{\left(b_{l}\right)} \boldsymbol{Q}_{j, F B}^{\left(b_{l}\right)}\right|^{2} \\
& \left.+2 \beta_{j k}^{\left(b_{i}, b_{l}\right)} \operatorname{Real}\left\{\boldsymbol{Q}_{j, F B}^{\left(b_{l}\right) \dagger} \boldsymbol{h}_{k}^{\left(b_{l}\right) \dagger} \boldsymbol{h}_{k}^{\left(b_{i}\right)} \boldsymbol{Q}_{j, F B}^{\left(b_{i}\right)}\right\}\right)
\end{aligned}
$$

hence,

$$
\begin{gathered}
E_{H, w}\left(\gamma_{k, F B}\right)=\sum_{\substack{j=1 \\
j \neq k}}^{K} E_{H, w}\left(\beta_{j k}^{\left(b_{i}, b_{i}\right)}\left|\boldsymbol{h}_{k}^{\left(b_{i}\right)} \boldsymbol{Q}_{j, F B}^{\left(b_{i}\right)}\right|^{2}\right) \\
+E_{H, w}\left(\beta_{j k}^{\left(b_{l}, b_{l}\right)}\left|\boldsymbol{h}_{k}^{\left(b_{l}\right)} \boldsymbol{Q}_{j, F B}^{\left(b_{l}\right)}\right|^{2}\right) \\
+2 E_{H, w}\left(\beta_{j k}^{\left(b_{i}, b_{l}\right)} \operatorname{Real}\left\{\boldsymbol{Q}_{j, F B}^{\left(b_{l}\right) \dagger} \boldsymbol{h}_{k}^{\left(b_{l}\right) \dagger} \boldsymbol{h}_{k}^{\left(b_{i}\right)} \boldsymbol{Q}_{j, F B}^{\left(b_{i}\right)}\right\}\right)
\end{gathered}
$$

And since $\boldsymbol{h}_{k}^{\left(b_{i}\right)}$ and $\boldsymbol{h}_{k}^{\left(b_{l}\right)}$ are mutually independent isotropically distributed random vectors in the vector space $\mathbb{C}^{N_{T}}$, having zero mean each, then

$$
E_{H, w}\left(\boldsymbol{Q}_{j, F B}^{\left(b_{l}\right) \dagger} \boldsymbol{h}_{k}^{\left(b_{l}\right) \dagger} \boldsymbol{h}_{k}^{\left(b_{i}\right)} \boldsymbol{Q}_{j, F B}^{\left(b_{i}\right)}\right)=0,
$$

therefore,

$$
E_{H, w}\left(\gamma_{k, F B}\right)=
$$

$$
\sum_{\substack{j=1 \\ j \neq k}}^{K} E_{H, w}\left(\beta_{j k}^{\left(b_{i}, b_{i}\right)}\left|\boldsymbol{h}_{k}^{\left(b_{i}\right)} \boldsymbol{Q}_{j, F B}^{\left(b_{i}\right)}\right|^{2}+\beta_{j k}^{\left(b_{l}, b_{l}\right)}\left|\boldsymbol{h}_{k}^{\left(b_{l}\right)} \boldsymbol{Q}_{j, F B}^{\left(b_{l}\right)}\right|^{2}\right)
$$

Since $E\left(\beta_{j k}^{\left(b_{i}, b_{i}\right)}\right)=E\left(\beta_{j k}^{\left(b_{l}, b_{l}\right)}\right)$, then 


$$
\begin{aligned}
E_{H, w}\left(\gamma_{k, F B}\right)= & (K-1) \cdot \overline{\beta_{j k}^{\left(b_{i}, b_{i}\right)}} \\
\cdot & E_{H, w}\left(\left|\boldsymbol{h}_{k}^{\left(b_{i}\right)} \boldsymbol{Q}_{j, F B}^{\left(b_{i}\right)}\right|^{2}+\left|\boldsymbol{h}_{k}^{\left(b_{l}\right)} \boldsymbol{Q}_{j, F B}^{\left(b_{l}\right)}\right|^{2}\right) \\
= & (K-1) \cdot \overline{\beta_{j k}^{\left(b_{i}, b_{i}\right)}} \cdot E_{H, w}\left(\left|\boldsymbol{H}_{k} \boldsymbol{Q}_{j, F B}\right|^{2}\right)
\end{aligned}
$$

where $\boldsymbol{H}_{k}=\left[\boldsymbol{h}_{k}^{(1)}, \ldots, \boldsymbol{h}_{k}^{(B)}\right]$ and $\boldsymbol{Q}_{k}=\left[\boldsymbol{Q}_{k}^{(1) \dagger}, \ldots, \boldsymbol{Q}_{k}^{(B) \dagger}\right]^{\dagger}$

Using the same argument as in the case of synchronous interference, $\overline{\boldsymbol{H}}_{k}$ can then be expressed as $\overline{\boldsymbol{H}}_{k}=\sqrt{1-d} \hat{\boldsymbol{H}}_{k}+$ $\sqrt{d} \boldsymbol{S}$. The expectation of the product of $\overline{\boldsymbol{H}}_{k}$ and $\boldsymbol{Q}_{j, F B}$ is then given by

$$
\begin{aligned}
E_{H, w}\left(\left|\overline{\boldsymbol{H}}_{k} \boldsymbol{Q}_{j, F B}\right|^{2}\right) & =E_{H, w}\left((1-d)\left|\hat{\boldsymbol{H}}_{k} \boldsymbol{Q}_{j, F B}\right|^{2}\right) \\
& +E_{H, w}\left(d\left|\boldsymbol{S} \boldsymbol{Q}_{j, F B}\right|^{2}\right)
\end{aligned}
$$

From the design criteria (7)

$$
\begin{gathered}
E_{H, w}\left(\sum_{\substack{j=1 \\
j \neq k}}^{K} \sum_{b_{i}=1}^{B} \sum_{b_{l}=1}^{B} \beta_{j k}^{\left(b_{i}, b_{l}\right)} \boldsymbol{Q}_{k}^{\left(b_{l}\right) \dagger} \hat{\boldsymbol{h}}_{j}^{\left(b_{l}\right) \dagger} \hat{\boldsymbol{h}}_{j}^{\left(b_{i}\right)} \boldsymbol{Q}_{k}^{\left(b_{i}\right)}\right)=0 \\
(K-1) \cdot \overline{\beta_{j k}^{\left(b_{i}, b_{i}\right)}} \cdot E_{H, w}\left(\left|\hat{\boldsymbol{H}}_{k} \boldsymbol{Q}_{j, F B}\right|^{2}\right)=0 \\
E_{H, w}\left(\left|\hat{\boldsymbol{H}}_{k} \boldsymbol{Q}_{j, F B}\right|^{2}\right)=0,
\end{gathered}
$$

then (28) reduces to

$$
E_{H, w}\left(\left|\overline{\boldsymbol{H}}_{k} \boldsymbol{Q}_{j, F B}\right|^{2}\right)=E_{H, w}(d) E_{H, w}\left(\left|\boldsymbol{S} \boldsymbol{Q}_{j, F B}\right|^{2}\right) .
$$

Since the term $\left|\boldsymbol{S} \boldsymbol{Q}_{j, F B}\right|^{2}$ is a beta $\left(1, B N_{T}-2\right)$ random variable, then its mean value is $\frac{1}{B N_{T}-1}$, and $E_{H, w}(d)=$ $1-\frac{1+N_{T}}{1+B N_{T}}\left(1-2^{\frac{-n}{N_{T}-1}}\right)$, then the rate loss in 10 becomes,

$$
\begin{aligned}
& \triangle R(P) \leq \log _{2}\left(1+(K-1) P \overline{\beta_{j k}^{\left(b_{i}, b_{i}\right)}} E_{H, w}\left(\left|\boldsymbol{H}_{k} \boldsymbol{Q}_{j, F B}\right|^{2}\right)\right) \\
& =\log _{2}\left(1+(K-1) P \overline{\beta_{j k}^{\left(b_{i}, b_{i}\right)}}\right. \\
& \left.N_{T} \sum_{b=1}^{B} L_{k}^{(b)} E_{H, w}\left(\left|\overline{\boldsymbol{H}}_{k} \boldsymbol{Q}_{j, F B}\right|^{2}\right)\right)
\end{aligned}
$$

therefore

$$
\begin{gathered}
\Delta R(P) \leq \log _{2}\left(1+(K-1) P \frac{\sum_{b=1}^{B} L_{k}^{(b)} N_{T}}{B N_{T}-1}\right. \\
\left.\cdot \overline{\beta_{j k}^{\left(b_{i}, b_{i}\right)}} \cdot\left(1-\frac{\left(1+N_{T}\right)}{\left(1+B N_{T}\right)}\left(1-2^{\frac{-n}{N_{T}-1}}\right)\right)\right),
\end{gathered}
$$

where the average value of $\beta_{j k}^{\left(b_{i}, b_{i}\right)}=\rho^{2}\left(\delta_{j k}^{\left(b_{1}\right)}\right)+\rho^{2}\left(\delta_{j k}^{\left(b_{1}\right)}-T_{s}\right)$. Using rectangular pulse shape with unit energy, $\rho\left(\delta_{j k}^{\left(b_{1}\right)}\right)=$ $\frac{1}{T_{s}}\left(T_{s}-\left|\delta_{j k}^{\left(b_{1}\right)}\right|\right)$. It can be easily shown that $E\left(\rho^{2}\left(\delta_{j k}^{\left(b_{1}\right)}\right)\right)=$ $E\left(\rho^{2}\left(\delta_{j k}^{\left(b_{1}\right)}-T_{s}\right)\right)=1 / 3$.

To conclude, the rate loss $\triangle R(P)$ given by 27 for the case of synchronous interference and (30) for the more general case of asynchronous interference shows the effect of quantizing the channel state information on the system performance.
It is clear from both equations, that the rate loss is an increasing function of the $\operatorname{SNR}(P)$. This means that the throughput of the limited feedback-based JLS system is bounded if the SNR is taken to infinity. In other words, the system with a fixed number of feedback bits is interferencelimited at high SNR.

It was shown in [29] that, in case of single-cell MIMO networks $B=1$, the rate loss tends to zero when the number of feedback bits grows to infinity. This same conclusion can be deduced from (27) and (30) by setting $B=1$ and $n \rightarrow \infty$. It was also shown that the number of feedback bits per mobile must be increased linearly with the SNR (in decibels) in order to achieve the full multiplexing gain. On the contrary, from (27) and 30 when $B \neq 1$, it is clear that the rate loss does not tend to zero when the number of feedback bits grows to infinity and subsequently increasing the number of bits in case of MUMIMO CoMP networks does not achieve the full multiplexing gain. This is due to the fact that optimizing the codeword choice for each BS channel separately does not imply that the global channel vector, which comprises the channels of all cooperating BSs for a certain user, is accurately quantized.

\section{Simulation Results}

To verify the analytical results, we simulate the downlink of an urban micro-cellular network using the 3GPP TR 25.996 channel model. The channel is assumed to be frequency selective and varying slowly in the time domain with a coherence time of $15 \mathrm{~ms}$, for a mobile station with a velocity of $10 \mathrm{~m} / \mathrm{sec}$. The system model consists of two cells, each with $1 \mathrm{BS}$ and $1 \mathrm{MS}$. The inter-BS distance is $500 \mathrm{~m}$. The MSs are uniformly distributed in a limited cell area around each BS from $-\pi / 3$ to $\pi / 3$ radians and at a distance that is at least $150 \mathrm{~m}$ from each BS. The path-loss coefficient for all the BS-MS channels is 2.0 (free-space propagation) up to a distance of $30 \mathrm{~m}$, and increases to 3.7 thereafter [30]. Using the MSs locations' distribution, described earlier and we consider $N_{T}=3, N_{R}=1, B=2$ and $K=2$.

The theoretical upper bound on the rate loss, given by (27), is compared to the simulated rate loss at different feedback bits in Fig 2. The simulated rate loss is lower than the upper bound primarily due to the use of Jensen's inequality. This result is repeated for the case of asynchronous interference, given by (30), in Fig 3. The rate loss in case of asynchronous environment is smaller than that in synchronous, the performance degradation in asynchronous case due to channel quantization is less. This is clear from the theoretical upper bounds given in 27 and 30 , where the term $\overline{\beta_{j k}^{\left(b_{i}, b_{i}\right)}}$ in the asynchronous rate loss upper bound is always less than or equal 1.

Another important result can be concluded from Fig. 4 and Fig 5, that is increasing the number of quantization bits does not result in a linear performance enhancement. This is clear when we compare the enhancement achieved when the number of quantization bits increases from 3 to 5 and the one achieved when the number of bits increases from 5 to 10 . Hence, increasing the number of feedback bits to infinity does not lead to a rate loss that tends to zero. This behavior is in contrast to the conventional single-cell MIMO case, where increasing the 


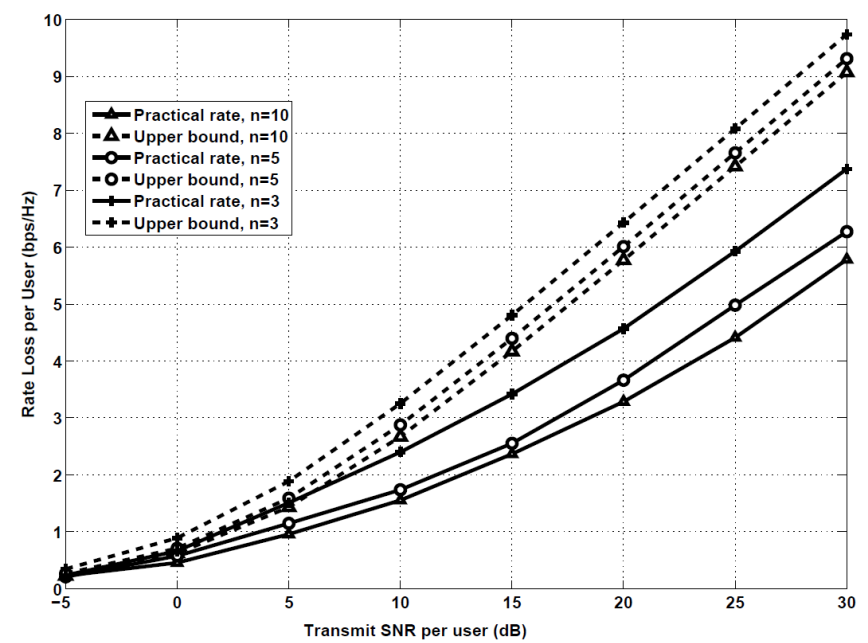

Fig. 2. Theoretical upper bound of rate loss and simulated one at 3, 5, and 10 feedback bits in an idealized synchronous environment.

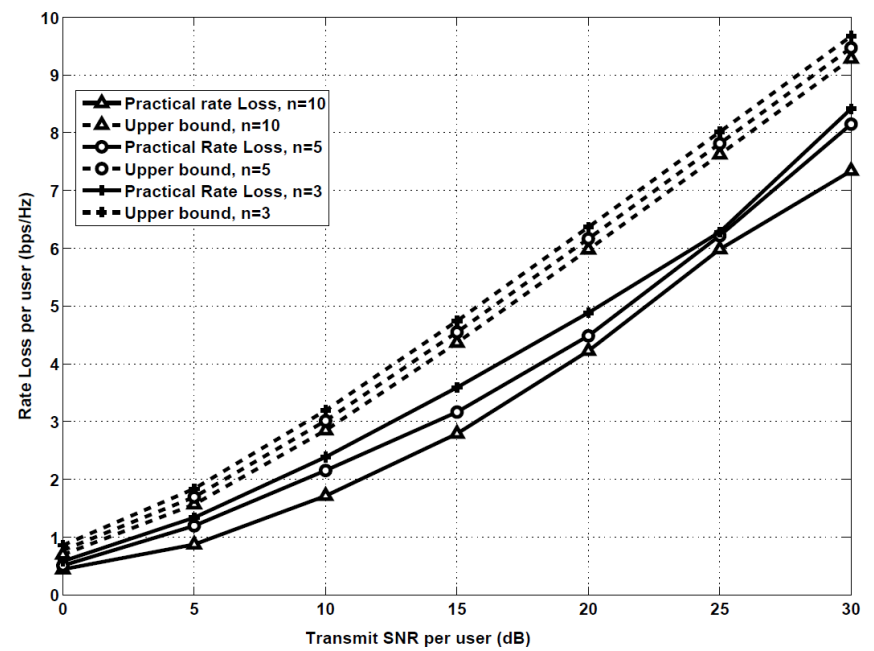

Fig. 3. Theoretical upper bound of rate loss and simulated one at 3, 5, and 10 feedback bits in asynchronous environment.

number of quantization bits to infinity guaranties a rate loss of zero.

\section{CONCLUSION}

In this paper we considered the finite rate feedback JLS precoder used in CoMP transmission as it is a suitable linear precoding scheme for the asynchronous interference case, that inevitably exists in the cooperative data transmission scenarios. We analyzed its performance in the case of finite capacity backhaul links. Random Vector Quantization has been used as the vector quantization technique to quantize the channel state. The rate loss due to this quantization is derived in both cases, the synchronous interference case and the asynchronous interference case. Finally simulation results verified the upper bounds derived for the case of finite feedback-JLS precoding scheme.

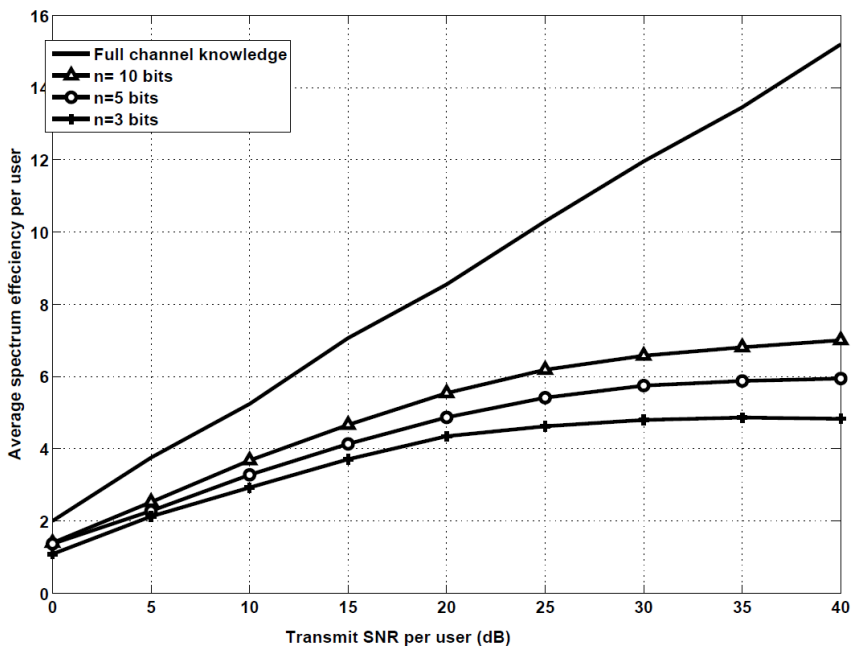

Fig. 4. Average Spectrum efficiency per user in case of using infinite channel feedback and finite feedback with 3,5 , and 10 feedback bits in an idealized synchronous environment.

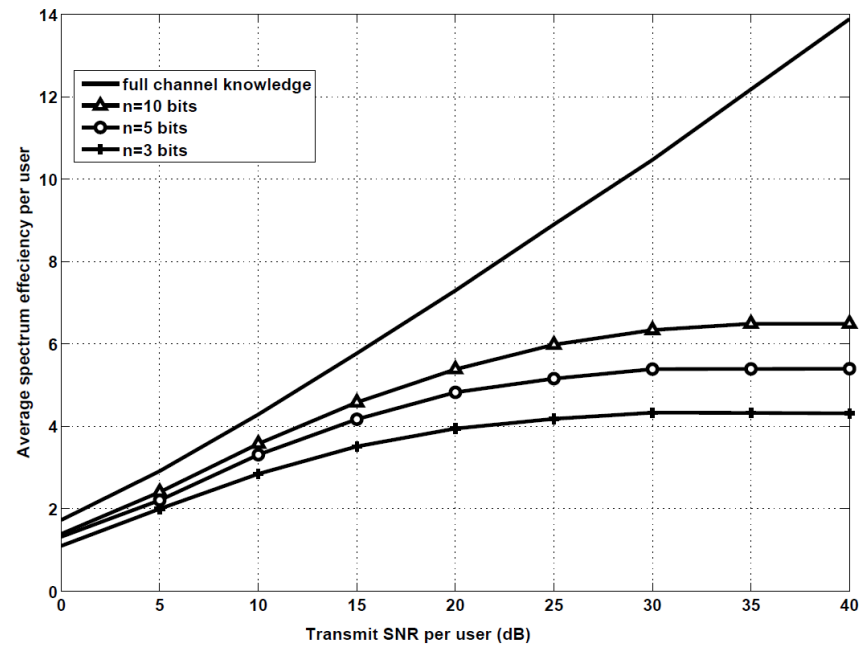

Fig. 5. Average Spectrum efficiency per user in case of using infinite channel feedback and finite feedback with 3, 5, and 10 feedback bits in asynchronous environment.

\section{REFERENCES}

[1] Technical Specification Group Radio Access Network; Evolved Universal Terrestrial Radio Access (E-UTRA); Further advancements for E-UTRA physical layer aspects, TR 36.814. (9.0.0), 3rd Generation Partnership Project (3GPP), March 2010.

[2] D. Lee et al., Coordinated multipoint transmission and reception in LTE-advanced: deployment scenarios and operational challenges, IEEE Communications Magazine, vol. 50, no. 2, pp. 148-155, Feb. 2012.

[3] M. Sawahashi, Y. Kishiyama, A. Morimoto, D. Nishikawa, and M. Tanno, Coordinated multipoint transmission/reception techniques for lteadvanced [coordinated and distributed MIMO], in Wireless Communications, IEEE, Vol.17, pp.26-34, June 2010.

[4] N. Jindal and A. Goldsmith, Dirty-paper coding versus TDMA for MIMO broadcast channels, Information Theory, IEEE Transactions on, Vol.51, pp.1783-1794, May 2005.

[5] S. Shamai and B. Zaidel, Enhancing the cellular downlink capacity via co-processing at the transmitting end, in Vehicular Technology Conference, 2001. VTC 2001 Spring. IEEE VTS 53rd, Vol. 3, pp. 1745 -1749 vol. 3, 2001.

[6] C. Windpassinger, R. Fischer, T. Vencel, and J. Huber, Precoding in 
multiantenna and multiuser communications, Wireless Communications, IEEE Transactions on, Vol. 3, pp. 1305 - 1316, July 2004.

[7] H. Dai, A. Molisch, and H. Poor, Downlink capacity of interferencelimited MIMO systems with joint detection, Wireless Communications, IEEE Transactions on, Vol. 3, pp. 442 - 453, March 2004.

[8] J. Lee and N. Jindal, High snr analysis for MIMO broadcast channels: Dirty paper coding versus linear precoding, Information Theory, IEEE Transactions on, Vol. 53, pp. 4787 -4792, Dec. 2007.

[9] O. Simeone, O. Somekh, Y. Bar-Ness, and U. Spagnolini, Uplink throughput of TDMA cellular systems with multicell processing and amplify-and-forward cooperation between mobiles, Wireless Communications, IEEE Transactions on, Vol. 6, pp. 2942-2951, Aug. 2007.

[10] D. Jaramillo-Ramrez, M. Kountouris and E. Hardouin, Coordinated Multi-Point Transmission With Imperfect CSI and Other-Cell Interference, IEEE Transactions on Wireless Communications, vol. 14, no. 4 pp. 1882-1896, April 2015.

[11] H. Bahrami and T. Le-Ngoc, A capacity achieving precoding scheme based on partial channel information for broadcast MIMO systems, in Wireless Communications and Networking Conference, 2007. WCNC 2007. IEEE, pp. 1045 -1050, March 2007.

[12] H. Zhang, N. Mehta, A. Molisch, J. Zhang, and H. Dai, Asynchronous interference mitigation in cooperative base station systems, Wireless Communications, IEEE Transactions on, Vol. 7, pp. 155 -165, Jan. 2008.

[13] J. Tang et al., Robust MMSE Design With Asynchronous Interference Mitigation in Cooperative Base Station Systems, Wireless Personal Communications, Volume 78, Issue 2, pp 889-903, Sep. 2014

[14] M. Sadek, A. Tarighat, and A. Sayed, A leakage-based precoding scheme for downlink multi-user MIMO channels, Wireless Communications, IEEE Transactions on, Vol. 6, pp. 1711 -1721, May 2007.

[15] R. Bhagavatula, R. Heath, and B. Rao, Limited feedback with join CSI quantization for multicell cooperative generalized eigenvector beamforming, in Acoustics Speech and Signal Processing (ICASSP), 2010 IEEE International Conference on, pp. 2838-2841, March 2010.

[16] R. Bhagavatula and R. Heath, Sum-rate maximizing beamforming in multicell systems with limited feedback, in Signals, Systems and Computers, 2009 Conference Record of the Forty-Third Asilomar Conference on, pp. 1838 -1842, Nov. 2009.

[17] http://planetmath. org/encyclopedia/RayleighQuotient. html

[18] D. J. Love and R. Heath, Feedback techniques for MIMO channels, in MIMO System Technology for Wireless Communications, pp. 113146, edition 1: CRC Press, March 2006.

[19] F. Boccardi, H. Huang, and M. Trivellato, Multiuser eigenmode transmission for MIMO broadcast channels with limited feedback, in Signal Processing Advances in Wireless Communications, 2007. SPAWC 2007. IEEE 8th Workshop on, pp. 1 -5, June 2007.

[20] N. Jindal, Antenna combining for the MIMO downlink channel, Wireless Communications, IEEE Transactions on, Vol. 7, pp. 3834 -3844, Oct. 2008.

[21] M. Trivellato, H. Huang, and F. Boccardi, Antenna combining and codebook design for the MIMO broadcast channel with limited feedback, in Signals, Systems and Computers, 2007. ACSSC 2007. Conference Record of the Forty-First Asilomar Conference on, pp. $302-308$, Nov. 2007.

[22] W. Santipach and M. Honig, Asymptotic performance of MIMO wireless channels with limited feedback, in Military Communications Conference, 2003. MILCOM 2003. IEEE, Vol. 1, pp. 141 - 146 Vol. 1, Oct. 2003.

[23] J. Roh and B. Rao, Transmit beamforming in multiple-antenna systems with finite rate feedback: a VQ-based approach, Information Theory IEEE Transactions on, Vol. 52, pp. 1101 -1112, March 2006.

[24] J. Conway, R. Hardin, and N. Sloane, Packing lines, planes, etc. Packings in grassmannian spaces, Experimental Mathematics, Vol. 5, no. 2, p. 139-159, 1996.

[25] C. K. Au-Yeung and D. J. Love, On the performance of random vector quantization limited feedback beamforming in a MISO system, Wireless Communications, IEEE Transactions on, Vol. 6, pp. 458-462, Feb. 2007.

[26] S. Jafar and A. Goldsmith, Isotropic fading vector broadcast channels:the scalar upper bound and loss in degrees of freedom, Information Theory, IEEE Transactions on, Vol. 51, pp. 848 - 857, March 2005.

[27] J.L.W.V. Jensen, Sur les fonctions convexes et les inégalités entre les valeurs moyennes, Acta Math., Vol. 30: 175, 1906.

[28] http://planetmath. org/encyclopedia/BetaRandomVariable. html

[29] M. Kountouris, R. de Francisco, D. Gesbert, D. Slock, and T. Salzer, Multiuser diversity-multiplexing tradeoff in MIMO broadcast channels with limited feedback, in Signals, Systems and Computers, 2006. ACSSC06. Fortieth Asilomar Conference on, pp. 364-368, Oct.29-Nov.1 2006.
[30] Technical Specification Group Radio Access Network; Spatial channel model for Multiple Input Multiple Output (MIMO) simulations, TR 25.996. (6.1.0), 3rd Generation Partnership Project(3GPP), Sept. 2003.

Yasmine Fahmy received the B. Sc with Honors from Cairo University in 1999 where she graduated as top of her class, the MSc. in 2001, and the $\mathrm{PhD}$. in 2005 , all in telecommunications, and all from the faculty of Engineering at Cairo University. She was appointed as a teaching assistant, at Cairo University, from September 1999 to September 2005. She is presently an associate professor at the Electronics and Communications Department Faculty of Engineering, Cairo University, Egypt. Her current field of interest includes wireless communication and modern coding techniques. Since 2009, she has been with the Center of Wireless Studies (CWS), Cairo University. 